\title{
ESPORAS TRILETES VERRUCOSAS DE LA FORMACIÓN LAGARCITO (ALBIANO?), SIERRA DE GUAYAGUAS, PROVINCIA DE SAN JUAN, ARGENTINA: ANÁLISIS BIOESTRATIGRÁFICO
}

\author{
NATALIA MEGO \& MERCEDES. B. PRÁMPARO \\ Instituto Argentino de Nivología, Glaciología y Ciencias Ambientales, Centro Científico Tecnológico, \\ CONICET, Av. Adrián Ruiz Leal s/n, Parque General San Martín, 5500 Mendoza, Argentina. \\ nmego@mendoza-conicet.gob.ar,mprampar@mendoza-conicet.gob.ar
}

\begin{abstract}
VERRUCATE TRILETE SPORES FROM THE LAGARCITO FORMATION (ALBIAN?) SIERRA DE GUAYAGUAS, SAN JUAN PROVINCE: ARGENTINA. BIOSTRATIGRAPHIC ANALYSIS. A detailed study of the systematics of 13 species of verrucate trilete spores recovered from the Lagarcito Formation is presented. Taxonomic studies of these types of spores with great morphological diversity are very important as they constitute a useful contribution for the clarification of many misidentifications usually very common within this morphologic group. The Lagarcito Formation is part of the El Gigante Group, formed by continental sediments of fluvio-deltaic origin deposited in an extensional basin. The material was collected in a section exposed in the eastern side of the Sierra de Guayaguas, southeast of the San Juan province and corresponds to shallow lacustrine sediments. The following species are described and illustrated: Concavissimisporites penolaensis Dettmann, Concavissimisporites punctatus (Delcourt \& Sprumont) Brenner, C. variverrucatus (Couper) Brenner, Concavissimisporites sp., Converrucosisporites cf. C. exquisitus Singh, Impardecispora apiverrucata (Couper) Venkatachala, Kar \& Raza, I. texensis Srivastava, I. sp., Leptolepidites major Couper, L. verrucatus Couper, Reyrea polymorphus Herngreen, Verrucosisporites rotundus Singh, $V$. varians Volkheimer. Within the palynoflora the verrucate trilete spores are abundant and correspond to the fern families: Lycopodiaceae, Selaginellaceae, Cyatheaceae, Osmundaceae, Dicksoniaceae and Schizaeaceae, indicative of local humid conditions near the lacustrine bodies. Based on the analysis of the occurrence and biostratigraphical value of the studied species in different Lower Cretaceous basins in Argentina and other related places, an Aptian-Albian range is concluded for the verrucate spore association recovered from the Lagarcito Formation. The present systematic study provides also new insight to the vegetal diversity developed during the Early Cretaceous in central-western Argentina.
\end{abstract}

Key words: trilete verrucate spores, Lagarcito Formation, Lower Cretaceous, Sierra de Guayaguas.

RESUMO - No presente estudo é apresentada a sistemática detalhada de 13 espécies de esporos triletes verrucosos recuperados da Formação Lagarcito. O estudo taxonômico deste tipo de esporos, cuja diversidade morfológica é vasta, é muito importante uma vez que contribui para o esclarecimento de dúvidas e erros de identificação muito comuns neste grupo morfológico. A Formação Lagarcito faz parte do Grupo El Gigante, formado por sedimentos continentais de origem fluvio-deltaica, depositados numa bacia extensional. O material analisado foi colhido numa seção exposta do lado oriental da Serra de Guayaguas, a Sudeste da Província de San Juan e corresponde a sedimentos lacustres pouco profundos. Várias espécies foram descritas e ilustradas: Concavissimisporites penolaensis Dettmann, C. punctatus (Delcourt \& Sprumont) Brenner, C. variverrucatus (Couper) Brenner, Concavissimisporites sp., Converrucosisporites cf. C. exquisitus Singh, Impardecispora apiverrucata (Couper) Venkatachala, Kar \& Raza, I. texensis Srivastava, Impardecispora sp., Leptolepidites major Couper, L. verrucatus Couper, Reyrea polymorphus Herngreen, Verrucosisporites rotundus Singh e V. varians Volkheimer. Os esporos triletes verrucosos são palinomorfos abundantes e correspondem às seguintes famílias de fetos: Lycopodiaceae, Selaginellaceae, Cyatheaceae, Osmundaceae, Dicksoniaceae e Schizaeaceae, cuja presença é indicativa de locais úmidos, próximos de corpos lacustres. Com base na análise de ocorrência e no valor bioestratigráfico das espécies estudadas em diferentes bacias argentinas do Cretáceo Inferior e outros lugares relacionados, foi possível reconhecer um intervalo Aptiano-Albiano para a associação de esporos verrucosos recuperados da Formação Lagarcito. O presente estudo fornece ainda uma nova perspectiva para a diversidade vegetal desenvolvida no Centro-Oeste da Argentina no início do Cretáceo.

Palavras-chave: esporos triletes verrucosos, Formação Lagarcito, Cretáceo Inferior, Sierra de Guayaguas.

\section{INTRODUCCIÓN}

La Formación Lagarcito, se encuentra bien expuesta en el lado este de la Sierra de Guayaguas, sureste de la Provincia de San Juan y en la Sierra de las Quijadas y del Gigante, en la Provincia de San Luis (Flores, 1969; Yrigoyen, 1975) y forma parte del Grupo del Gigante (Rivarola \& Spalletti, 2006) correspondiente a la Cuenca de San Luis. En la Sierra de Guayaguas (NW de la Cuenca de San Luis), en la sección denominada "La Yesera Sur", la Formación Lagarcito está 
integrada por una sucesión psamopelítica-evaporítica rojiza con intercalaciones de yeso y anhidrita portadora de una importante palinoflora de origen continental. Dichos sedimentos fueron depositados en una cuenca de tipo extensional y corresponden a un ambiente de planicie aluvial con canales fluviales efímeros y lagos o lagunas someras. Ocasionalmente estos lagos fueron rellenados por depósitos evaporíticos endorreicos relacionados lateralmente con areniscas eólicas (Chiappe et al., 1998). La Formación Lagarcito presenta registros de ambientes lacustres y fluviales a través de sus depósitos. Los ambientes lacustres varían de un lago poco profundo, meromíctico de baja salinidad a somero estratificado, llegando a depósitos salinos de tipo sabkha (Prámparo et al., 2005) (Figura 1). El contenido paleontológico de la Formación Lagarcito conocido hasta el momento comprende restos de pterosaurios, peces, conchóstracos, plantas y una gran variedad de trazas de invertebrados (Bonaparte, 1970; Chiappe et al., 1998) provenientes del perfil Loma del Pterodaustro en la Sierra de Las Quijadas, en la Provincia de San Luis. Por otra parte la Formación La Cantera, bien expuesta en la Sierra del Gigante (sector SE de la cuenca de San Luis) y correspondiente a la secuencia basal dentro del Grupo del Gigante, fue asignada por su contenido palinológico al Aptiano tardío (Prámparo 1990, 1994; Prámparo et al., 2007). La Formación Lagarcito corresponde a la secuencia superior dentro del mismo grupo (Rivarola \& Spalletti, 2006). En base a su contenido fosilífero y a las relaciones estratigráficas Chiappe et al. (1998), asignaron a la Formación Lagarcito tentativamente al Albiano. Hasta el momento sin embargo, no hay dataciones absolutas de esta formación.

Los primeros resultados de un estudio paleontológico conjunto (algas, conchóstracos y ostrácodos) de la sección "La Yesera Sur" en la Sierra de Guayaguas, fueron dados a conocer en Prámparo et al. (2005). Ninguno de los grupos fósiles estudiados aportó datos conclusivos sobre la edad de la formación, por lo que los autores sugirieron mantener la edad albiana propuesta por Chiappe et al. (1998). De los niveles fértiles recuperados en la Formación Lagarcito, en la Sierra de Guayaguas, se obtuvo una microflora muy bien conservada y variada, constituida principalmente por granos de polen de gimnospermas y esporas de pteridofitas y briofitas. No se encontraron granos de polen de Angiospermas.

El objetivo de esta contribución es presentar un estudio sistemático de detalle de las esporas trilete verrucosas recuperadas de la Formación Lagarcito, y analizar su distribución en otras microfloras de la misma edad de Argentina y áreas relacionadas, con la finalidad de: (i) interpretar su valor bioestratigráfico dentro de las asociaciones del Cretácico Temprano y (ii) poder de esta manera en base a los rangos de distribución de las especies contribuir a definir con mayor precisión la edad de la Formación Lagarcito. El grupo morfológico de esporas verrucosas registra una amplia presencia en las distintas cuencas cretácicas del mundo, sin embargo aún es poco reconocido su valor bioestratigráfico, tal como ocurre generalmente con todos los grupos de palinomorfos continentales, debido a que en general su distribución puede estar asociada a factores ecológicos locales.
El presente estudio sistemático de detalle de este grupo de esporas trilete, constituye un aporte fundamental para conocer la diversidad vegetal existente durante el Cretácico Temprano, en la región centro oeste de Argentina. Los resultados aquí obtenidos son un complemento a los estudios que se vienen realizando dentro de la misma cuenca (Cuenca de San Luis), pero en otro depocentro (Sierra del Gigante), sobre la macro y microflora de la Formación La Cantera (Prámparo, 1988, 1989, 1994; Puebla, 2009; Puebla et al., 2012) ya que ambas unidades (formaciones La Cantera y Lagarcito) pertenecen al Grupo El Gigante.

\section{MATERIAL Y MÉTODOS}

Un total de 19 muestras de la sección La Yesera Sur, lado este de la Sierra de Guayaguas, fueron colectadas (M. Prámparo y J.P. Milana) y cinco de ellas resultaron fértiles para palinomorfos. Todas las muestras fueron procesadas según las técnicas físico-químicas convencionales de extracción (HCl-HF) (Volkheimer \& Melendi, 1976), filtradas con filtro metálico de $10 \mu \mathrm{m}$ teñidas con safranina y finalmente montadas con gelatina-glicerina. Los preparados resultantes están almacenados con los números de catalogo 5861, 5862, 5967, 5969, 6595 en la Paleopalinoteca del Instituto Argentino de Nivologia, Glaciología y Ciencias Ambientales (IANIGLA) bajo la sigla MPLP (MendozaPaleopalinoteca- Laboratorio de Paleopalinologia) del CCT (Centro Científico Tecnólogico), Mendoza, Argentina. El estudio de los preparados y el fotografiado de los mismos fueron realizados con un microscopio Olympus BX50, con una cámara digital Olympus adaptada. Las coordenadas del material ilustrado corresponden a England Finder.

El mapa de ubicación del área de estudio y un detalle de la litología de donde se obtuvieron las muestras palinológicas puede verse en la Figura 1. La sección completa, perfil "La Yesera Sur" fue levantado por J.P. Milana en la Sierra de Guayaguas y está publicado en Prámparo et al. (2005).

\section{SISTEMÁTICA PALINOLÓGICA}

La clasificación de las esporas dispersas estudiadas (géneros y especies fósiles) se realizó siguiendo el Código Internacional de Nomenclatura para Algas, Hongos y Plantas (Melbourne Code, McNeill et al., 2012) a partir de su probable afinidad botánica. Este criterio es seguido y fundamentado ampliamente por numerosos autores, entre ellos Fensome (1987) quien discute el uso de métodos artificiales de clasificación (Potonié \& Kremp, 1954; Potonié, 1956) y explica las dificultades que presenta especialmente cuando se intenta trabajar con conceptos biológicos. Se agrupan en la presente contribución a las diferentes especies de esporas verrucosas recuperadas de la Formación Lagarcito, según las categorías superiores de Licofitas y Monilofitas (Pryer et al., 2004; Smith et al., 2006) y dentro de los grupos se presenta a los taxones en orden alfabético. Se describen las esporas, tratando de sintetizar en cada género las características básicas para su diagnosis. Se discute su distribución principalmente 


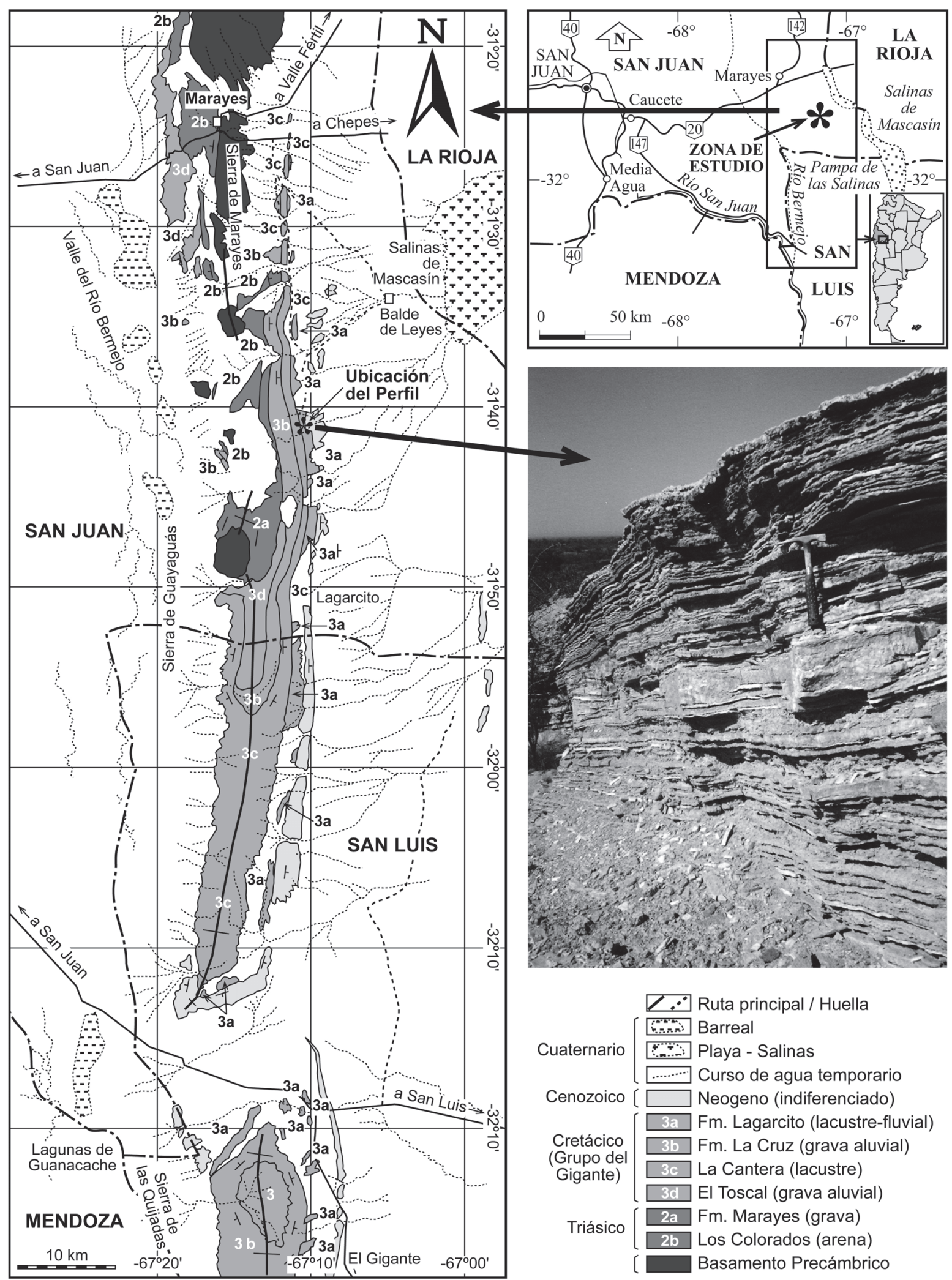

Figura 1. Mapa de ubicación de la sección muestreada de la Formación Lagarcito en la Sierrra de Guayaguas, sureste de la Provincia de San Juan. A la derecha foto de detalle de la litología predominante en la Formación Lagarcito (evaporitas con intercalaciones de arcilitas). Las muestras palinológicas fueron tomadas de los niveles pelíticos intercalados en el yeso.

Figure 1. Location map of the studied section of the Lagarcito Formation in the Sierra de Guayaguas, southeast of the San Juan Province. At the right, photograph of the evaporitic lithology of Lagarcito Formation. The palynological samples were taken from the pelites interbedded within the gypsum. 
en el Hemisferio Sur, con un especial énfasis en su registro en Sudamérica y Argentina.

\section{Esporas dispersas de Licofitas y Monilofitas}

\section{Concavissimisporites (Delcourt \& Sprumont) Delcourt, Dettmann \& Hughes, 1963}

Este género según la enmienda de Delcourt et al. (1963), incluye esporas trilete con escultura verrucosa uniformemente distribuida, y lados cóncavos a casi rectos. La diagnosis genérica ha sido levemente alterada por Singh (1964) para incluir esporas con escultura subgranulosa a granulosa. Fensome (1987) realiza una amplia y completa discusión sobre los géneros triangulares con verrugas basado en la historia sistemática de los mismos. Es así que dicho autor amplía la diagnosis del género Concavissimisporites para incluir esporas triangulares con una mayor concentración y tamaño de la ornamentación en los ápices. Propone considerar a Concavissimisporites un sinónimo de los géneros Tuberositriletes e Impardecispora y resalta la restricción de este género a esporas con ornamentación compuesta por elementos individuales y la exclusión de especies con desarrollo de muros apicales. Uno de los fundamentos de su discusión es el grado de superposición que observó en las descripciones y diagnosis de estos taxones. Si bien es cierto que en algunas asociaciones se observa una gradación en la ornamentación de los individuos con ornamentación verrucosa, preferimos seguir aquí el criterio dado por Burden \& Hills (1989) de mantener el genero Conncavissimisporites separado de Impardecispora.

Especie tipo. Concavissimisporites verrucosus Delcourt \& Sprumont, 1955.

\section{Concavissimisporites penolaensis Dettmann, 1963} (Figuras 2A-B)

Material. 5967: P41/4, O44/0 MPLP; 5967 C: R38/4 MPLP; 5968 I: E26/0 MPLP; 5968B: Q33/0 MPLP, P42/3 MPLP.

Dimensiones. 49-88 $\mu \mathrm{m}$ (seis ejemplares).

Observaciones. Esta especie se caracteriza por poseer una ornamentación constituida por verrugas (menos de $1 \mu \mathrm{m}$ de alto) de bases poligonales densamente dispuestas en ambas caras de la espora. Algunos de los ejemplares de la Formación Lagarcito presentan una lesura delimitada por un margo y en otros, los rayos de la lesura terminan en una bifurcación (curvatura imperfecta), características que no han sido mencionadas en la descripción original de la especie dada por Dettmann (1963) ni tampoco en la realizada por Llorens (2008) para los primeros ejemplares registrados en la Argentina, sin embargo estos caracteres pueden observarse con claridad en el material ilustrado por dichos autores.

Distribución. Valanginiano-Albiano del sureste de Australia (Dettmann, 1963). En Argentina ha sido mencionada en el Aptiano, Formación Punta del Barco, Cuenca Baqueró (Macizo del Deseado) (Llorens, 2008); y en Patagonia extra-andina, Formación Cañadón Calcáreo (Valanginiano?) Volkheimer et al. (2009).

\author{
Concavissimisporites punctatus \\ (Delcourt \& Sprumont) Brenner, 1963 \\ (Figura 2C)
}

Material estudiado. 5861F: E37/4 MPLP.

Dimensiones. $65 \mu \mathrm{m}$ (un ejemplar).

Observaciones. Esta especie se caracteriza por poseer una delicada ornamentación subgranulosa, la cual como menciona Srivastava (1975) puede fusionarse con los elementos vecinos para formar rúgulas, rasgo que se observa en el único ejemplar registrado en la Formación Lagarcito.

Distribución. En el Hemisferio Sur se registró en: Sur de África, Jurásico Tardío-Cretácico Temprano (Barremiano) (Scott, 1976); Sur de Chile, Hauteriviano (Cranwell \& Srivastava, 2009). En Argentina esta especie fue registrada en la Formación Kachaike, Albiano de la Provincia de Santa Cruz (Archangelsky et al., 2012) y en la Formación Huincul (Albiano?/Cenomaniano), Cuenca Neuquina (Vallati, 2001).

\section{Concavissimisporites variverrucatus \\ (Couper) Brenner, 1963 \\ (Figuras 2D-E)}

Material. 5861 D: T38/0 MPLP; 5968A: E44/3 MPLP.

Dimensiones. $73 \mu \mathrm{m}$ (dos ejemplares).

Observaciones. La ornamentación constituida solo por verrugas es el rasgo distintivo de esta especie. De acuerdo con Singh (1964) las mismas presentan dimensiones de 1-5 $\mu \mathrm{m}$, con forma y espaciado de los elementos verrucosos muy variable entre los distintos especímenes. Los ejemplares de la Formación Lagarcito presentan verrugas (1-2 $\mu \mathrm{m}$ de alto) y también un patrón finamente micropuntuado.

Distribución. En el Hemisferio Sur se conocen registros en: Sur de Chile, Hauteriviano (Cranwell \& Srivastava, 2009), Nordeste de Brasil, (Cuenca del Rio do Peixe) Jurásico terminal-Neocomiano (Lima \& Coelho, 1987); Valanginiano-Aptiano de la Plataforma continental de Alagoas, Sergipe y cuencas de Sergipe/Alagoas y Reconcavo/Tucano (Regali et al., 1974; Regali \& Viana, 1989). En Argentina: Formación Springhill, ValanginianoBarremiano (Quattrocchio et al., 2006).

\section{Concavissimisporites $\mathrm{sp}$.}

(Figura 2F)

Material. 5861C: Q21/2 MPLP.

Dimensiones. $61 \mu \mathrm{m}$ (un ejemplar).

Descripción. Espora trilete, contorno triangular con lados levemente cóncavos y ápices redondeados. Lesura simple, recta, rayos de la lesura extendiéndose $2 / 3$ del radio de la espora. Exina de espesor uniforme $(2 \mu \mathrm{m})$, ornamentada por verrugas redondeadas (1,5 $\mu \mathrm{m}$ de alto y $1-2 \mu \mathrm{m}$ de ancho). Verrugas de forma irregular, algunas se fusionan determinando un patrón rugulado. 

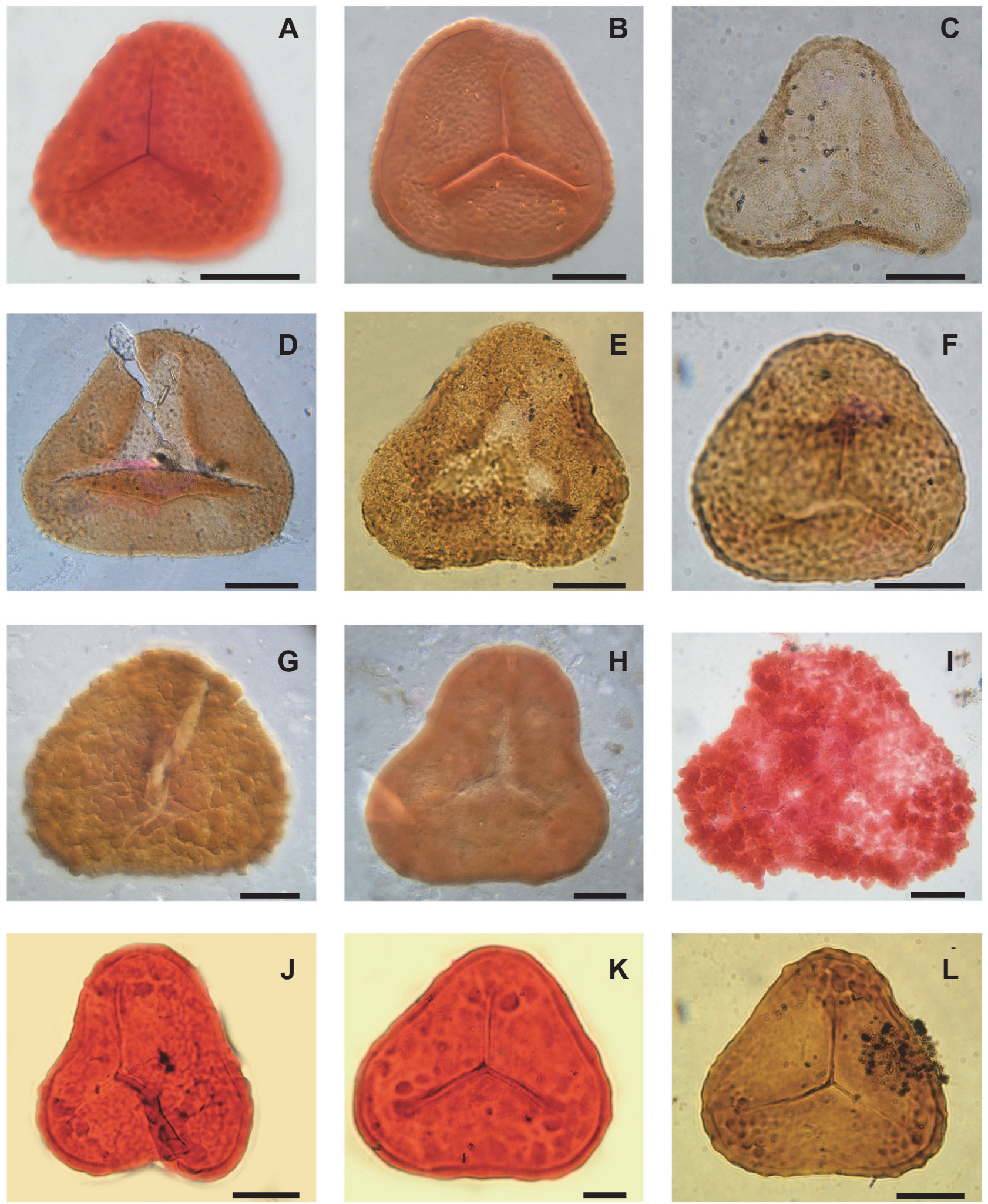

Figura 2/ Figure 2. Esporas verrucosas/ Verrucate spores. A-B, Concavissimisporites penolaensis Dettmann. A, MPLP5968I: E26/0 vista proximal/ proximal view; B, MPLP 5967C: R38/4 vista proximal/ proximal view, foco intermédios/ intermediate focus. C, Concavissimisporites punctatus (Delcourt \& Sprumont) Brenner, MPLP 5861F: E37/4. D-E, Concavissimisporites variverrucatus (Couper) Brenner. D, MPLP 5968A: E44/3; E, MPLP5861 D: T38/0. F, Concavissimisporites sp., MPLP 5861C: Q21/2. G, Converrucosisporites cf. C. exquisitus Singh, MPLP 5968X: M24/0. H-J, Impardecispora apiverrucata (Couper) Venkatachala, Kar \& Raza. H, MPLP 5968 Z: Q41/0; I, MPLP 5967I: G36/1; J, MPLP 5968K: C41/3. K-L, Impardecispora texensis Srivastava. K, MPLP 5967: U40/3; L, MPLP5861 E: K27/4. Escalas/ Scale bars: $\mathrm{A}-\mathrm{J}=20 \mu \mathrm{m} ; \mathrm{K}-\mathrm{L}=10 \mu \mathrm{m}$. 
Observaciones. La característica principal de esta especie es su patrón de ornamentación verrucoso a rugulado. Concavissimisporites variverrucatus presenta una distribución uniforme de las verrugas. Hay un gran parecido entre el espécimen de la Formación Lagarcito y Concavissimisporites sp. A descripto por Llorens (2008) pero este último material presenta menores dimensiones $(30,8-40 \mu \mathrm{m})$. C. irroratus Paden Phillips \& Félix, 1971 es también similar al material estudiado pero de acuerdo a dichos autores los elementos esculturales nunca se fusionan. C. penolaensis difiere por poseer verrugas bajas con bases poligonales.

\section{Converrucosisporites Potonié \& Kremp, 1954}

Este género incluye esporas triletes triangulares a subtriangulares con escultura verrucosa densamente dispuesta. Difiere de Verrucosisporites Ibrahim emend. Smith \& Butterworth, 1967 porque este último género posee un contorno circular a subcircular.

Especie tipo. Converrucosisporites triquetrus (Ibrahim) Potonié \& Kremp, 1954.

\section{Converrucosisporites cf. C. exquisitus Singh, 1971}

(Figura $2 \mathrm{G}$ )

Material. 5868X: M24/0 MPLP.

Dimensiones. $89 \mu \mathrm{m}$ (un ejemplar)

Descripción. Espora trilete, contorno triangular con lados rectos a levemente convexos y ápices redondeados. Lesura simple, recta, los rayos de la lesura se extienden a aproximadamente $2 / 3$ del radio de la espora. Exina ornamentada por verrugas de forma irregular (aproximadamente de $1,5 \mu \mathrm{m}$ de alto), uniformemente distribuidas, algunas se fusionan determinando un patrón rugulado.

Observaciones. El $c f$. se refiere al menor tamaño de las verrugas en comparación a los elementos ornamentales en el material de Singh (1971) (6 a $10 \mu \mathrm{m}$ ), además el ejemplar de la Formación Lagarcito posee un diámetro ecuatorial ligeramente mayor que el dado por Singh $(68-84 \mu \mathrm{m})$.

\section{Impardecispora Venkatachala, Kar \& Raza, 1968}

La separación y distinción entre los géneros Impardecispora, Trilobosporites y Concavissimisporites es de gran utilidad para realizar una sistemática y bioestratigrafía precisa (Srivastava, 1975). Impardecispora Venkatachala, Kar \& Raza se diferencia de Trilobosporites Pant ex R. Potonié por carecer de valvas, y de Concavissimisporites Delcourt \& Sprumont emend. Delcourt, Dettmann \& Hughes por tener una ornamentación granulosaverrucosa desigualmente distribuida.

Trilobosporites incluye esporas trilete con contorno triangular, valvas ornamentadas en los ápices y exina granular a verrucosa. Concavissimisporites se diferencia por poseer una exina más homogéneamente ornamentada en ambas caras. Según Dörhöfer \& Norris (1977), existen estrechas gradaciones morfológicas entre las diferentes especies correspondientes a los morfogéneros Trilobosporites y Concavissimisporites. Dichos autores se refieren a las especies más antiguas del género Trilobosporites que aparecen en el Berriasiano temprano, caracterizadas por su gran tamaño y por poseer valvas muy pronunciadas, que se van reduciendo en las formas más nuevas y en algunos casos se dividen. Ambas variaciones morfológicas se desarrollaron independientemente una de la otra entre sí. Las especies del género Concavissimisporites en cambio mostrarían una tendencia morfológica de poco a marcadamente cóncavas, de las formas más antiguas a las más modernas. Al mismo tiempo aparecen esporas que poseen una escultura densa en las áreas apicales en vez de poseer valvas verdaderas. Esta es una nueva característica que evolucionó del linaje de Concavissimisporites o de la línea de Trilobosporites con valvas reducidas. Venkatachala et al. (1968) asignaron estas esporas con escultura densa en las áreas apicales al género Impardecispora. En el material de la Formación Lagarcito no se encontraron formas valvadas asignables al género Trilobosporites según los criterios aceptados en este trabajo y explicados anteriormente siguiendo autores como Venkatachala et al. (1968) o Dörhöfer \& Norris (1977); se han recuperado en cambio una gran variedad de formas asignables a los géneros Concavissimisporites e Impardecispora. La presencia de estos géneros en el Cretácico Inferior de otras formaciones y otras cuencas de Argentina es constante, sobre todo teniendo en cuenta los criterios de separación de los géneros antes mencionados. De este modo, algunos especímenes asignados a distintas especies del género Trilobosporites como $T$. apiverrucatus o T. trioreticulosus de la Cuenca Austral (Medina et al., 2008; Archangelsky et al., 2012) corresponderían en realidad al género Impardecispora por tratarse de formas sin valvas. Por lo tanto, es importante reevaluar la importancia bioestratigráfica de estos géneros y especies en el Cretácico Inferior de Argentina a partir de su correcta identificación.

Especie tipo. Impardecispora apiverrucata Venkatachala, Kar \& Raza, 1968.

\section{Impardecispora apiverrucata (Couper) Venkatachala, Kar \& Raza, 1968}

(Figuras 2H-J)

Ver sinonimia completa en Srivastava (1975, p. 44).

En Argentina:

1966 Trilobosporites apiverrucatus Archangelsky \& Gamerro, p. 235, fig. 1.

2008 Trilobosporites apiverrucatus Archangelsky \& Gamerro. Medina, Archangelsky, Guler, Archangelsky \& Cárdenas, p. 280, fig. C.

2012 Concavissimisporites apiverrucatus (Archangelsky \& Gamerro). Archangelsky, Archangelsky \& Cladera, p. 33, fig. D.

Material. 5967 I: G36/1; 5968 K: C41/3; 5968 Z: Q41/0 MPLP.

Dimensiones. 74-92 $\mu \mathrm{m}$ (tres ejemplares).

Observaciones. Los tres ejemplares de la Formación Lagarcito asignados a Impardecispora apiverrucata presentan contorno triangular, lados marcadamente cóncavos a rectos. 
Lesura recta, extendiéndose 3/4 del radio de la espora con margo y exina ornamentada proximal y distalmente por pequeñas verrugas $(1-2 \mu \mathrm{m})$. La ornamentación apical es más conspicua $(2-5 \mu \mathrm{m})$ y está densamente dispuesta.

Distribución. En el Hemisferio Sur: Cranwell \& Srivastava (2009) Hauteriviano del Sur de Chile (Impardecispora apiverrucata); Argentina: Cuenca Austral, Berriasiano temprano-Aptiano temprano (Archangelsky et al., 1984), formaciones Kachaike (Baldoni et al., 2001; Archangelsky et al., 2012) y formaciones Kachaike y Piedra Clavada, Aptiano tardío-Albiano temprano (Medina et al., 2008), Grupo Baqueró, Barremiano-Aptiano (Archangelsky \& Gamerro, 1966).

\section{Impardecispora texensis Srivastava, 1975}

(Figuras 2K-L)

Material. 5861 E: K27/4 MPLP; 5968 J: D26/0 MPLP; 5967: U40/3 MPLP.

Dimensiones. 59-74 $\mu \mathrm{m}$ (tres ejemplares).

Descripción. Espora trilete, contorno triangular, lados rectos a cóncavos, ápices redondeados. Lesura simple, recta, extendiéndose aproximadamente $2 / 3$ del radio de la espora. Exina gruesa, se encuentra ornamentada por verrugas (2-3 $\mu \mathrm{m}$ de alto, $2 \mu \mathrm{m}$ de ancho) las mismas son de mayor tamaño y se encuentran más densamente dispuestas en los ápices y en la superficie distal.

Observaciones. Impardecispora texensis se diferencia de I. purverulenta por presentar verrugas individuales en los ápices. Dettmann (1963) y Padden Phillips \& Felix (1971) hacen mención para Trilobosporites purverulentus sobre la presencia de un retículo en los ápices formado por la fusión de verrugas y hasta la presencia de una ornamentación foveolada, es decir una escultura negativa, característica que no se observa en los ejemplares de la Formación Lagarcito por lo cual se los asignó a I. texensis.

Distribución. No hay registros de esta especie en Argentina y Sudamérica.

\section{Impardecispora sp.}

(Figuras 3A-C)

\section{Material. 5861E: G31/1 MPLP; 5967 D28/2 MPLP. \\ Dimensiones. $75-80 \mu \mathrm{m}$ (dos ejemplares).}

Descripción. Espora trilete, contorno triangular con lados rectos a levemente cóncavos, ápices redondeados. Lesura recta, extendiéndose $2 / 3$ del radio de la espora, con presencia de un margo. Exina gruesa con un espesor constante en toda la espora de 3 a $4 \mu \mathrm{m}$. Cara distal ornamentada con verrugas circulares (1-3 $\mu \mathrm{m}$ de diámetro) distribuidas en forma regular. La cara proximal es lisa excepto en la región apical donde se observa una ornamentación constituida por verrugas en forma de gota $(10 \mu \mathrm{m}$ de largo y $8 \mu \mathrm{m}$ de ancho), circunscriptas exclusivamente a los extremos de la lesura (Figuras 3B,C).

Observaciones. Esta especie se distingue de las otras descriptas en la literatura, por el tipo de ornamentación apical de su cara proximal asociada a los extremos de la lesura.
Leptolepidites Couper, 1953

Especie tipo. Leptolepidites verrucatus Couper, 1953.

Leptolepidites major Couper, 1958

(Figura 3D)

Material. 5861B: R19/4 MPLP; 5968O: V31/0 MPLP.

Dimensiones. 77-81 $\mu \mathrm{m}$ (dos ejemplares).

Observaciones. Los ejemplares de la Formación Lagarcito tienen un diámetro mayor que los típicos dados para la especie $(36-51 \mu \mathrm{m})$ con una superficie proximal lisa, y una superficie distal ornamentada por verrugas de tamaño variable, en forma de domo y densamente dispuestas (2-5 $\mu \mathrm{m}$ de altura; $2-4 \mu \mathrm{m}$ de ancho). Esta especie se diferencia de Leptolepidites verrucatus porque esta última es de dimensiones menores y contorno más subtriangular. En la Formación La Cantera, perteneciente a la misma cuenca (Cuenca de San Luis), Prámparo (1988) describió e ilustró un único ejemplar al que asignó a $L$. major pero de menores dimensiones $(55 \mu \mathrm{m})$ y con un pobre estado de preservación al presentar corrosión en las verrugas.

Distribución. Ampliamente distribuida en sedimentos mesozoicos del mundo. En el Hemisferio Sur: Nordeste de Brasil (Cuenca del Rio do Peixe), Jurásico terminal -Albiano (Regali et al., 1974; Lima \& Coelho, 1987; Regali \& Viana, 1989; Coimbra et al., 2002). En Argentina: Grupo Baqueró, Barremiano-Aptiano (Archangelsky \& Villar de Seoane, 1994; Del Fueyo et al., 2007); Cuenca del Macizo del Deseado. Formación Huitrín (Barremiano-Albiano), Cuenca Neuquina (Volkheimer \& Salas, 1976) y en la Formación La Cantera (Aptiano tardío), Cuenca de San Luis (Prámparo, 1988, 1994).

\section{Leptolepidites verrucatus Couper, 1953}

(Figuras 3E-G)

\section{Material. 5968A: F36/0 MPLP; 59680: X38/1 MPLP.}

Dimensiones. $38 \mu \mathrm{m}$ (dos ejemplares).

Observaciones. Los ejemplares de la Formación Lagarcito presentan una lesura sinuosa, extendiéndose hasta el borde interno de la zona ecuatorial formada por la fusión de verrugas ( $5 \mu \mathrm{m}$ de alto; 5-7 $\mu \mathrm{m}$ de ancho). Rayos de la lesura acompañados por labios (1-1, $5 \mu \mathrm{m}$ de ancho total). Superficie proximal lisa. Superficie distal ornamentada por verrugas conspicuas (7-9 $\mu \mathrm{m}$ de alto; 6-7 $\mu \mathrm{m}$ de ancho).

Distribución. Nueva Zelanda (Couper, 1953); Australia (Burger, 1988; Dettmann, 1963). Sur de África, Jurásico Tardío-Cretácico Temprano (Barremiano) (Scott, 1976), Nordeste de Brasil, Jurásico Tardío-Hauteriviano (Lima \& Coelho, 1987); Região de Marabá, Estado do Pará, Brasil, Aptiano (Lima et al., 1980); Formación Castellanos, Uruguay, Albiano (Campos et al., 1998). En Argentina: formaciones Anfiteatro de Ticó (Del Fueyo et al., 2007); y Kachaike, Aptiano tardío-Albiano temprano (Baldoni et al., 2001). 
Reyrea Herngreen, 1973

Especie tipo. Reyrea polymorphus Herngreen, 1973.

\section{Reyrea polymorphus Herngreen, 1973}

(Figura 3H)

Material. 5967 C: T38/1

Dimensiones. $62 \mu \mathrm{m}$ (un ejemplar).

Descripción. Espora trilete, contorno oval, una de las caras con una ornamentación más reducida y compuesta por verrugas chatas, mientras que la otra cara se encuentra ornamentada por elementos esculturales conspicuos, algunos de ellos dispuestos uno encima del otro, circulares y otros presentan una base ensanchada y terminan con una especie de verruga sobreimpuesta, distribuidos en forma aleatoria, sin seguir ningún tipo de patrón, abiertamente dispuestos y de tamaños muy variables, 3-11 $\mu$ m (diámetro basal), y 7-11 $\mu \mathrm{m}$ de alto. Exina gruesa $(3 \mu \mathrm{m})$.

Observaciones. Este espécimen se distingue del género Uvaesporites porque este último presenta un contorno subtriangular, es proximalmente liso con una ornamentación principalmente compuesta por elementos en forma de uva o gema y una marca trilete prominente, extendida hasta el ecuador. Se diferencia del género Leptolepidites porque este presenta una ornamentación constituida por proyecciones verrucosas.

Distribución. Aptiano-Albiano de Egipto (Mahmoud et al., 2007), Aptiano-Albiano de Ghana (Atta Peters \& Salami, 2006), Albiano de Brasil (Herngreen, 1973). Esta es la primera mención de este taxón en el Cretácico de Argentina.

Verrucosisporites Ibrahim emend. Smith \& Butterworth, 1967

Este género incluye esporas trilete con contorno circular a subcircular y exina cubierta en ambas caras por verrugas aproximadamente redondeadas, de base ancha y densamente dispuestas.

Especie tipo. Verrucosisporites verrucosus (Ibrahim) Ibrahim, 1933.

Verrucosisporites rotundus Singh, 1964

(Figuras 3I-J)

Material. 5861B: U39/2 MPLP; 5968B: N32/4 MPLP; 5969: N36/3 MPLP; 5969: D46/0 MPLP; 6595 2: R33/0 MPLP; 6595 3: L31/0 MPLP.

Dimensiones. 54-90 $\mu \mathrm{m}$ (seis ejemplares).

Observaciones. Esta especie se caracteriza por su contorno circular y por poseer una ornamentación constituida por verrugas redondeadas y regularmente dispuestas en ambas caras. Los ejemplares registrados en la Formación Lagarcito poseen mayores dimensiones que aquellos de la descripción original $(45-50 \mu \mathrm{m})$ con una exina ornamentada en ambas superficies por verrugas redondeadas (3-4 $\mu \mathrm{m}$ de alto, 3-6 $\mu \mathrm{m}$ de ancho), apretadamente dispuestas.
Distribución. No hay registros de esta especie en Argentina y Sudamérica. Verrucosisporites rotundus ha sido reportada en África para el Aptiano-Albiano del noroeste de Egipto y Sudan (Schrank \& Ibrahim, 1995; Eisawi et al., 2012).

Verrucosisporites varians Volkheimer, 1972

(Figura 3K)

Material. 5861B: K21/1 MPLP; 5861C: R23/0, M40/3 MPLP; 5861D: O27/1 MPLP; 5861F: F27/0, M34/4 MPLP; 5967A: G31/3 MPLP; 5967B: E42/3, R40/1 MPLP; 5968Z: F25/4 MPLP.

Dimensiones. $52-102 \mu \mathrm{m}$ (10 ejemplares)

Observaciones. Verrucosisporites varians se distingue por presentar una gran variabilidad de tipos de elementos esculturales y por la densidad de la ornamentación. Los ejemplares aquí estudiados presentan una ornamentación distribuida en ambas caras en forma homogénea, constituida mayormente por verrugas (3-5 $\mu \mathrm{m}$ de alto, 3-5 $\mu \mathrm{m}$ de ancho), gemas, báculas y en menor proporción granos y conos. El único ejemplar ilustrado por Prámparo (1988) de la Formación La Cantera, mide $57 \mu \mathrm{m}$ y presenta una variabilidad de elementos ornamentales y una distribución de los mismos muy similar a los observados en las esporas estudiadas de la Formación Lagarcito.

Distribución. En Argentina: de amplia distribución en el Jurásico de la Cuenca Neuquina. Formación Lajas, Caloviano de Neuquén (Volkheimer, 1972), Formación Chacai-Có, Pliensbaquiano (Volkheimer, 1974), Lias de Paso Flores (Volkheimer et al., 1981), Bayociano de Formación Cura Niyeu (Gonzalez-Amicón \& Volkheimer, 1982). En el Cretácico, en la Formación La Cantera, Aptiano tardío, Cuenca de San Luis (Prámparo 1988); formaciones Springhill y Pampa Rincón, Valanginiano superior-Cenomaniano (Quattrocchio et al., 2006).

\section{ANÁLISIS BIOESTRATIGRÁFICO}

La asociación de esporas verrucosas estudiadas en esta contribución está compuesta mayormente por taxones con un amplio rango estratigráfico pero con un acmé durante el Cretácico. Algunos de los taxones seleccionados por su importancia desde el punto de vista bioestratigráfico son: Concavissimisporites penolaensis, C. punctatus, C. variverrucatus, Impardecispora texensis, I. (Trilobosporites) apiverrucata, Reyrea polymorphus y Verrucosisporites rotundus.

En Australia, Concavissimisporites penolaensis fue recuperada en secciones de la Cuenca Otway (Dettmann, 1963), y pertenece a la Asociación Coptospora paradoxa, la cual en base a información paleontológica fue asignada al Aptiano-Albiano inferior a superior. Esta especie es considerada por dicha autora como estratigráficamente significativa dentro de la Asociación Coptospora paradoxa.

Impardecispora texensis ha sido descripta para el Grupo Fredericksburg en el sur de Estados Unidos (Srivastava, 1975, 1981) y es por su rango restringido un buen taxón guía del Albiano, en el hemisferio norte. El registro en la Formación Lagarcito es el primero de esta especie en Argentina. 

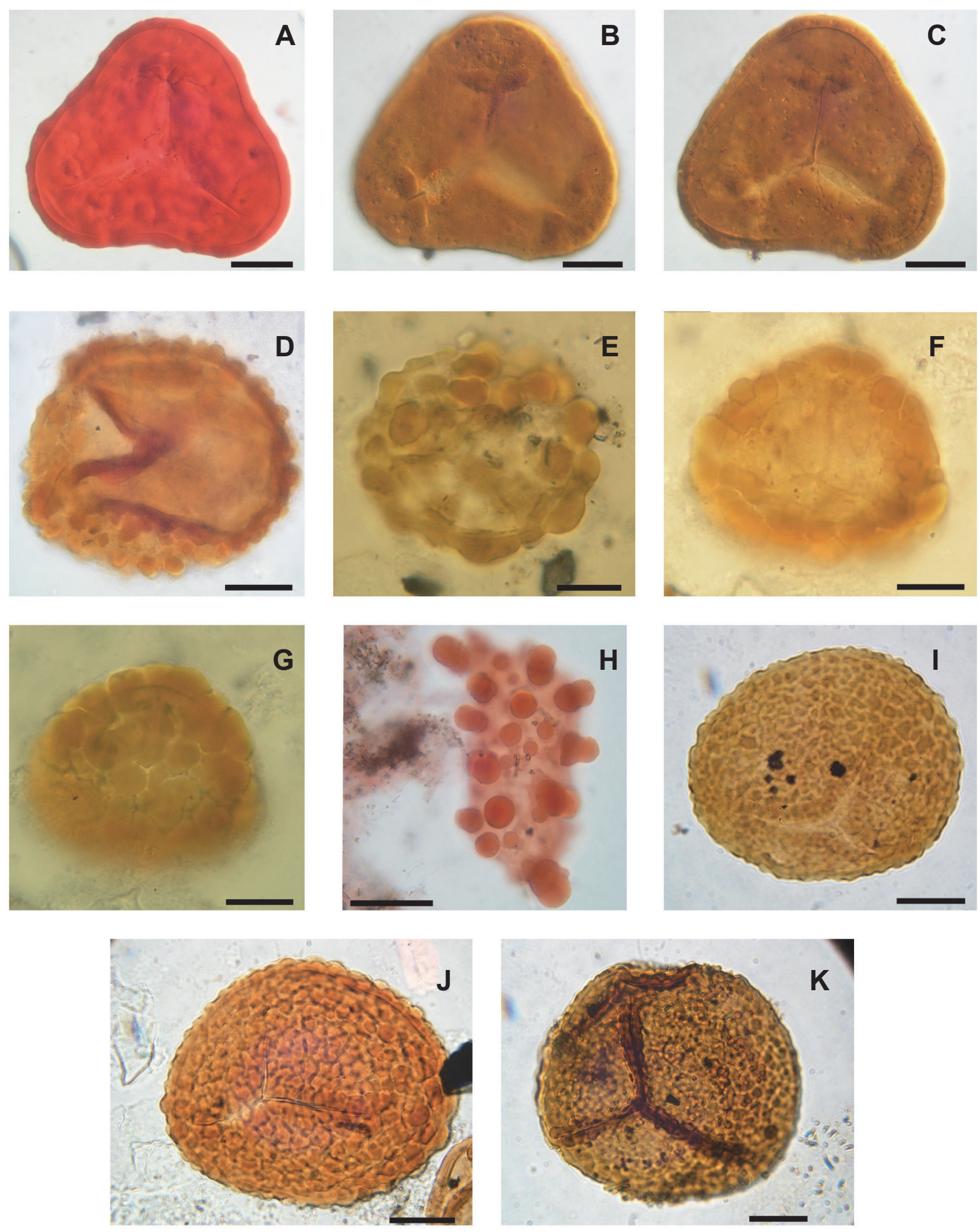

Figura 3/ Figure 3. Esporas verrucosas/ Verrucate spores. A-C, Impardecispora sp. A, MPLP5967 E33/1; B-C, MPLP 5861E: G31/1. D, Leptolepidites major Couper, MPLP 5968O: V31/0. E-G, Leptolepidites verrucatus Couper. E, MPLP 5968A: F36/0; F, MPLP5968O: X38/1; G, MPLP5968O: X38/1. H, Reyrea polymorphus Herngreen, MPLP5967 C: T38/1. I-J, Verrucosisporites rotundus Singh. I, MPLP 6595 2: R33/0; J, MPLP 5968B: N32/4. K, Verrucosisporites varians Volkheimer, MPLP 5861F: M34/4. Escalas/ Scale bars: A-C, E-K = $10 \mu \mathrm{m} ; \mathrm{D}=20 \mu \mathrm{m}$. 
Concavissimisporites variverrucatus es un elemento frecuente $(40 \%)$ de la Zona I (Barremiano-Aptiano) y poco frecuente en la Zona II (Albiano) correspondientes al Grupo Potomac, Canadá. C. punctatus también es frecuente en la Zona I pero no exclusiva de la misma (Brenner, 1963). Impardecispora (Trilobosporites) apiverrucata y C. punctatus son consideradas especies indicadoras de edad albiana para Canadá (Singh, 1964; Playford, 1971). I. (Trilobosporites) apiverrucata se ha registrado en sedimentos del Jurásico Tardío-Albiano del mundo (Srivastava, 1975).

En Brasil, Concavissimisporites variverrucatus aparece en el Barremiano tardío en diversas cuencas del Noreste. Un ejemplo es el de la Cuenca de Tucano donde $C$. variverrucatus junto a Retimonocolpites peroreticulatus, Afropollis operculatus, A. zonatus y Stellatopollis barghoornii hacen su aparición justo debajo del tope de la Zona de Stellatopollis bituberensis, que caracteriza la base del Aptiano temprano (Regali \& Viana, 1989). C. variverrucatus está presente tanto en la Zona de Aequitriradites spinulosus como en la Zona de Tucanopollis crisopolensis en varias cuencas del Noreste de Brasil (Regali et al., 1974; Regali \& Viana, 1989).

Recientemente Eisawi et al. (2012) reconocieron seis zonas-asociación informales para Sudan, Cuenca de Muglad en el Norte de África, basadas en la presencia y abundancia de taxones de miosporas seleccionados (index taxa) para un intervalo que va desde el Cretácico Inferior al Paleoceno. Definieron el tope de la Zona-Asociación II (Barremiano-Aptiano) por la última aparición de Psilosisporites trichopapillosus y la ocurrencia entre otros taxones de Concavissimisporites punctatus y $C$. cf. $C$. variverrucatus. Por otra parte, definieron la Zona-Asociación III (Aptiano-Albiano) por la última ocurrencia entre otros taxones, de Verrucosisporites rotundus. Este último taxón ya había sido también reportado para el Aptiano-Albiano del noroeste de Egipto (Schrank \& Ibrahim, 1995).

Finalmente, es de destacar la primera mención de Reyrea polymorphus para Argentina, este es un importante taxón guía para el Aptiano-Albiano de Egipto (Mahmoud et al., 2007) y Ghana (Atta Peters \& Salami, 2006); y para el Albiano de Brasil (Herngreen, 1973).

Se analizó por otra parte, la presencia de los taxones identificados en la Sierra de Guayaguas (Formación Lagarcito), en las diferentes asociaciones microflorísticas publicadas del Cretácico Inferior de Argentina, especialmente aquellas unidades con un rango de edad entre el AptianoAlbiano, organizadas de norte a sur, para tratar de encontrar el valor bioestratigráfico de las especies, en el sur de Sudamérica.

En el norte de Argentina, la única palinoflora estudiada de esta edad es la perteneciente a la Cuenca del Grupo Salta, Formación La Yesera (Albiano-Cenomaniano) (Narváez, 2009). Las formaciones La Yesera y Lagarcito comparten especies principalmente del grupo de las cicatricosas pertenecientes a la familia Anemiaceae (Narváez et al., 2013) pero ninguna especie dentro del grupo morfológico de esporas trilete verrucosas.

En la región del centro-oeste de Argentina se ubica la Cuenca de San Luis (entre 31 a $33^{\circ}$ de latitud sur), cuyos principales depocentros (Sierra del Gigante y Sierra de Las Quijadas, Provincia de San Luis y Sierra de Guayaguas, Provincia de San Juan) se encuentran representados por los sedimentos continentales del Grupo del Gigante (Flores \& Criado-Roque, 1972). La Formación La Cantera, (estratigráficamente ubicada por debajo de la Formación Lagarcito) en la Sierra del Gigante en San Luis, fue asignada por su contenido palinológico al Aptiano tardío (Prámparo, 1989, 1990, 1994, 1999; Prámparo et al., 2007) por la presencia de granos de polen de angiospermas. La misma ha aportado una importante y diversa asociación palinológica con un predominio de formas acuáticas (algas de agua dulce) y con escasas esporas trilete. La Formación Lagarcito comparte con la Formación La Cantera solamente: Leptolepidites major y Verrucosisporites varians (Tabla 1).

En el sur de Argentina, en Patagonia, El Grupo Baqueró, asignado por dataciones radiométricas $(206 \mathrm{~Pb} / 238 \mathrm{U}$ edad $114.67 \pm 0.18 \mathrm{Ma}$ ) al Aptiano tardío (Limarino et al., 2012) presenta en común con nuestra asociación las especies Concavissimisporites penolaensis, Leptolepidites major e Impardecispora (Trilobosporites) apiverrucata.

Impardecispora (Trilobosporites) apiverrucata es uno de los taxones que caracterizan la palinozona InterulobitesForaminisporis, de las cuatro definidas por Archangelsky et al. (1984) para el Sur de Argentina, de edad Valanginiana superior a Hauteriviana superior. En esta zona hace su aparición por primera vez en Argentina este taxón.

Barreda \& Archangelsky (2006) y Archangelsky et al. (2008) registraron una asociación palinológica con la presencia de polen de angiospermas tropicales (Schrankipollis, Pennipollis y la primera aparición de Walkeripollis (Winteraceae) en la Formación Kachaike, Provincia de Santa Cruz. La edad estimada de la Formación Kachaike es albiana-cenomaniana en base a palinomorfos marinos (dinoflagelados) y amonites (Guler \& Archangelsky, 2006; Aguirre-Urreta, 2002). Las formaciones Lagarcito y Kachaike comparten tres especies del grupo morfológico de las esporas verrucosas: Concavissimisporites punctatus, Leptolepidites verrucatus e Impardecispora (Trilobosporites) apiverrucata (Tabla 1). Finalmente, en la misma cuenca, la Formación Piedra Clavada (Albiano), en la Provincia de Santa Cruz (Archangelsky et al., 2008) comparte con la Formación Lagarcito los siguientes taxones del grupo morfológico de las verrucosas: L. major y L. verrucatus.

\section{CONSIDERACIONES FINALES}

De los niveles fértiles recuperados en la Formación Lagarcito, en la Sierra de Guayaguas, se obtuvo una microflora muy bien conservada y variada, en cuanto a cantidad de especies, constituida principalmente por granos de polen de gimnospermas pertenecientes a efedráceas (Ephedripites spp.) y cheirolepidiaceas (Classopollis spp.). En la asociación total estudiada, las esporas trilete del tipo verrucosas, constituyen un $4-8 \%$ del total y corresponderían a las familias de pteridofitas: Lycopodiaceae, Selaginellaceae, Cyatheaceae, 
Tabla 1. Distribución de las especies con importancia bioestratigráfica, del grupo de esporas triletes verrucosas, en las distintas cuencas del Cretácico Inferior de Argentina.

Table 1. Species distribution of verrucate trilete spores with biostratigraphic importance, in different Lower Cretaceous basins of Argentina.

\begin{tabular}{|c|c|c|c|c|c|}
\hline \multirow{2}{*}{ Taxa } & \multicolumn{2}{|c|}{ Cuenca de San Luis } & \multicolumn{2}{|c|}{ Cuenca Austral } & \multirow{2}{*}{$\begin{array}{c}\text { Cuenca } \\
\text { Baqueró } \\
\text { Grupo Baqueró }\end{array}$} \\
\hline & Formación Lagarcito & Formación La Cantera & $\begin{array}{c}\text { Formación } \\
\text { Piedra Clavada }\end{array}$ & $\begin{array}{c}\text { Formación } \\
\text { Kachaike }\end{array}$ & \\
\hline Concavissimisporites penolaensis & $\mathrm{X}$ & & & & $\mathrm{X}$ \\
\hline Concavissimisporites punctatus & $\mathrm{X}$ & & & $\mathrm{X}$ & \\
\hline Concavissimisporites variverrucatus & $\mathrm{X}$ & & & & \\
\hline Leptolepidites major & $\mathrm{X}$ & $\mathrm{X}$ & $\mathrm{X}$ & & $\mathrm{X}$ \\
\hline Leptolepidites verrucatus & $\mathrm{X}$ & & $\mathrm{X}$ & $\mathrm{X}$ & \\
\hline Impardecispora (Trilobosporites) apiverrucata & $\mathrm{X}$ & & & $\mathrm{X}$ & $\mathrm{X}$ \\
\hline Impardecispora texensis & $\mathrm{X}$ & & & & \\
\hline Verrucosisporites rotundus & $\mathrm{X}$ & & & & \\
\hline Verrucosisporites varians & $\mathrm{X}$ & $\mathrm{X}$ & & & \\
\hline
\end{tabular}

Osmundaceae, Dicksoniaceae y Schizaeaceae (Dettmann, 1963; Srivastava, 1975), las cuales se habrían desarrollado en un ambiente local húmedo cercano a los cuerpos lacustres.

De acuerdo a lo anteriormente expuesto y analizando los biocrones de las especies seleccionadas que se muestran en la Tabla 2 se puede inferir un rango de distribución especialmente concentrado en el intervalo Aptiano-Albiano. En el caso del género Concavissimisporites sólo algunas especies llegan hasta el Cenomaniano (C. punctatus) pero en general es poco común su ocurrencia en asociaciones del Cretácico Superior. Impardecispora texensis es un buen fósil guía para el Albiano del Hemisferio norte; lo mismo ocurre con Verrucosisporites rotundus que es un indicador del Aptiano-Albiano del norte de África y Reyrea polymorphus, un buen indicador del Aptiano-Albiano del norte de Africa y del Albiano de Brasil. La ocurrencia de estas especies en la Formación Lagarcito constituye el primer registro en Argentina y podrían ser consideradas para la distinción de zonas palinológicas en un futuro, especialmente en las cuencas del Centro-Oeste argentino. Si bien los datos obtenidos no son concluyentes, se puede indicar con bastante certeza un rango Aptiano-Albiano para la asociación analizada de esporas verrucosas según su presencia en las diferentes cuencas cretácicas de Argentina, demostrando así el valor bioestratigráfico de varios taxones de este grupo.

Tabla 2. Rango de distribución de taxones en el ámbito de Gondwana seleccionados por su importancia bioestratigráfica, identificados en la Formación Lagarcito. Impardecispora texensis, por ser la primera mención en el Hemisferio Sur, su rango de distribución corresponde a EUA.

Table 2. Gondwanic distribution range chart of taxa identified in the Lagarcito Formation, selected by their biostratigraphic value. As Impardecispora texensis is mentioned for the first time for the Southern Hemisphere, its distribution range corresponds to USA.

\begin{tabular}{|c|c|c|c|c|c|c|c|c|c|c|c|c|c|}
\hline \multirow{3}{*}{ Periodo-Edad } & \multirow{3}{*}{ Jurásico } & \multicolumn{12}{|c|}{ Cretácico } \\
\hline & & \multicolumn{6}{|c|}{ Temprano } & \multicolumn{6}{|c|}{ Tardío } \\
\hline & & Berr & Val & Hau & Barr & Apt & Alb & Cen & Tur & Con & San & Cam & Maas \\
\hline \multicolumn{14}{|l|}{ Concavissimisporites penolaensis } \\
\hline \multicolumn{14}{|l|}{ Concavissimisporites variverrucatus } \\
\hline \multicolumn{14}{|l|}{ Concavissimisporites punctatus } \\
\hline \multicolumn{14}{|l|}{ Impardecispora texensis } \\
\hline \multicolumn{14}{|l|}{ Impardecispora (Trilobosporites) apiverrucata } \\
\hline \multicolumn{14}{|l|}{ Reyrea polymorphus } \\
\hline Verrucosisporites rotundus & & & & & & & & & & & & & \\
\hline
\end{tabular}




\section{AGRADECIMIENTOS}

Las autoras desean agradecer a J.P. Milana (UNSJ) por la colaboración en la toma de muestras y levantamiento del perfil sedimentológico; a A. Moschetti (IANIGLA) por el tratamiento de las muestras palinológicas, a R. Bottero (IANIGLA) por la realización de los gráficos y a Sarita Camacho, Universidade do Algarve, Portugal, por la traducción del resumen al portugués. Este trabajo fue financiado parcialmente por el Consejo Nacional de Investigaciones Científicas y Técnicas de la Argentina (CONICET). Finalmente, a la editora M.J. Garcia y a las revisoras A. Archangelsky y E.P. Ferreira por sus valiosas contribuciones para mejorar el manuscrito.

\section{REFERENCIAS}

Aguirre-Urreta, M.B. 2002. Invertebrados del Cretácico Inferior. In: M.J. Haller (ed.), Geología y Recursos Naturales de Santa Cruz. CONGRESO GEOLÓGICO ARGENTINO, XV, Relatorio, El Calafate, p. 439-459.

Archangelsky, S. \& Gamerro, J.C. 1966. Estudio palinológico de la Formación Baqueró (Cretácico), provincia de Santa Cruz. III. Ameghiniana, 4:229-236.

Archansgelsky, S. \& Villar de Seoane, L. 1994. Estudios palinológicos de la Formación Baqueró (Cretácico), Provincia de Santa Cruz, Argentina. VI. Ameghiniana, 31:41-53.

Archangelsky, S.; Archangelsky, A. \& Cladera, G. 2012. Palinología y paleoambientes en el perfil de Bajo Comisión (Cretácico), provincia de Santa Cruz, Argentina. Revista del Museo Argentino de Ciencias Naturales, 14:23-39.

Archangelsky A.; Archangelsky S.; Poiré, D.G. \& Canessa, N.D. 2008. Registros palinológicos de la Formación Piedra Clavada (Albiano) en su área tipo, provincia de Santa Cruz, Argentina. Revista del Museo Argentino de Ciencias Naturales, 10:185-198.

Archangelsky, S.; Baldoni, A.; Gamerro, J.C. \& Seiler, J. 1984. Palinologia estratigráfica del Cretácico de Argentina Austral. III. Distribución de las especies y conclusiones. Ameghiniana, 21:15-33.

Atta Peters, D. \& Salami, M.B. 2006. Aptian-Maastrichtian palynomorphs from the offshore Tano Basin, western Ghana. Journal of African Earth Sciences, 46:379-394. doi: 10.1016/j. jafrearsci.2006.07.002

Baldoni, A.M.; Askin, R.A. \& Ragona, D. 2001. Palynology of the Lower Cretaceous Kachaike Formation, Santa Cruz province, Argentina. In: INTERNATIONAL PALYNOLOGICAL CONGRESS, 9, 2001. Proceedings, Houston, Texas, USA, p. 191-200.

Barreda, V. \& Archangelsky, S. 2006. The southernmost record of tropical pollen grains in the Cretaceous of Patagonia, Argentina. Cretaceous Research, 27:778-787. doi:10.1016/j. cretres.2006.02.002

Bonaparte, J.F. 1970. Pterodaustro guiñazui gen. et sp. nov. Pterosaurio de la Formación Lagarcito, provincia de San Luis, Argentina y su significado en la geología regional (Pterodactylidae). Acta Geológica Lilloana, 10:207-226.

Brenner, G.J. 1963. The spores and pollen of the Potomac Group of Maryland. Bull. Maryland Department of Geology, Bulletin Mines and Water Resources, 27:1-215.

Burden, E.T. \& Hills, L.V. 1989. Illustrated key to the genera of Lower Cretaceous terrestrial palynomorphs (excluding megaspores) of Western Canada. AASP Contributions Series, 21:1-147.
Burger, D. 1988. Early Cretaceous environments in the Eromanga Basin; palynological evidence from GSQ Wyandra-1 corehole. In: P.A. Jell \& G. Playford (eds.) Palynological and Palaeobotanical Studies in Honour of Basil E. Balme. Association of Australasian Palaeontologists, Sydney, p. 173-186 (Memoir 5).

Campos, C.C.; García, M.J.; Dino, R.; Veroslavsky, G.; Saad, A.R. \& Fulfaro, V.J. 1998. Palinomorfos da Formação Castellanos, na porção norte da Bacia de Santa Lucía, Albiano do Uruguai. Revista da Universidade Guarulhos, Geociencias, 3:5-21.

Chiappe, L.; Rivarola, D.; Cione, A.; Frenegal-Martinez, M.; Sozzi, H.; Buatois, L.; Gallego, O.; Laza, J.; Romero, E.; Lopez Arbarello, A.; Buscalioni, A.; Marsicano, C.; Adamonis, S.; Ortega, F.; Mcgeehe, S. \& Di Iorio, O. 1998. Biotic association and paleoenvironmental reconstruction of the "Loma del Pterodaustro" fossil site (Lagarcito Formation, Early Cretaceous, San Luis, Argentina). Geobios, 31:349-369. doi: 10.1016/S00166995(98)80018-1

Coimbra, J.C.; Arai, M. \& Carreño, A.L. 2002. Biostratigraphy of Lower Cretaceous microfossils from the Araripe Basin, northeastern Brazil. Geobios 35:687-698. doi: 10.1016/S00166995(02)00082-7

Couper, R.A. 1953. Upper Mesozoic and Cainozoic spores and pollen grains from New Zealand. New Zealand Geological Survey, Palynology Bulletin, 22:1-77.

Couper, R.A. 1958. British Mesozoic microspores and pollen grains. Palaeontographica, 108B:75-179.

Cranwell, L.M. \& Srivastava, S.K. 2009. An Early Cretaceous (Hauterivian) spore-pollen assemblage from Southern Chile. Palynology, 33:241-280. doi:10.2113/gspalynol.33.1.241.

Delcourt, A.F. \& Sprumont, G. 1955. Les spores et grains de pollen du Wealdien du Hainault. Liège, Société Géologique de Belgique, 73 p. (Mémoire 4).

Delcourt, A.F.; Dettmann, M.E. \& Hughes, N.F. 1963. Revision of some Lower Cretaceous microspores from Belgium. Palaeontology, 6:42-45.

Del Fueyo, G.; Villar de Seoane, L.; Archangelsky, A.; Guler, V.; Llorens, M.; Archangelsky, S.; Gamerro, J.C.; Musacchio, E.A.; Passalia, M.G. \& Barreda, V.D. 2007. Biodiversidad de las Paleofloras de Patagonia Austral durante el Cretácico Inferior. In: S. Archangelsky; T. Sanchez \& E.P. Tonni (eds.) Asociación Paleontológica Argentina, Buenos Aires, p. 101-122 (Publicación Especial 11).

Dettmann, M.E. 1963. Upper Mesozoic microfloras from SouthEastern Australia. Proceedings of the Royal Society of Victoria, 77:1-148.

Dörhöfer, G. \& Norris, G. 1977. Discrimination and correlation of highest Jurassic and lowest Cretaceous terrestrial palynofloras in north-west Europe. Palynology, 1:79-93. doi: 10.1080/01916122.1977.9989151

Eisawi, A.A.M.; Ibrahim, A.B.; Rahim, O.B.A. \& Schrank, E. 2012. Palynozonation of the Cretaceous to Lower Paleogene strata of the Muglad Basin, Sudan. Palynology, 36:191-207. doi: 10.1080/01916122.2011.633634

Fensome, R.A. 1987. Taxonomy and biostratigraphy of schizeaealan spores from the Jurassic-Cretaceous boundary beds of the Aklavik Range, District of Mackenzie. Palaeontographica Canadiana, 4:1-49.

Flores, M.A. 1969. El Bolsón de las Salinas de la Provincia de San Luis. In: JORNADAS GEOLÓGICAS ARGENTINAS, 4, 1969. Actas, Mendoza, v. 1, p. 311-327.

Flores, M.A. \& Criado-Roque, P. 1972. Cuenca de San Luis. In: A. Leanza (ed.), Geologia Regional Argentina. Academia Nacional de Ciencias, Cordoba, p. 567-580. 
González-Amicón, O.R. \& Volkheimer, W. 1982. Palinología estratigráfica del Jurásico de Chacai-Co y adyacencias (Cuenca Neuquina, República Argentina). 3: Descripciones sistemáticas de los palinomorfos de la Formación Cura Niyeu (Bayociano). Ameghiniana, 19:165-178.

Guler, M.V. \& Archangelsky, S. 2006. Albian dinoflagellate cysts from the Kachaike Formation, Austral Basin, Southwest Argentina. Revista del Museo Argentino de Ciencias Naturales, 8:179-184.

Herngreen, G.F.W. 1973. Palynology of Albian-Cenomanian strata of Borehole 1-QS-1-MA, State of Maranhão, Brazil. Pollen et Spores, 15:515-555.

Ibrahim, A.C. 1933. Sporenformen des Aegirhorizonts des Ruhrreviers. Wurzburg, University of Berlin Konrad Triltsch, 47 p.

Lima, M.R. \& Coelho, M.P.C.A. 1987. Estudo palinológico da sondagem estratigráfica de Lagoa do Forno, Bacia do Rio do Peixe, Cretáceo do Nordeste do Brasil. Boletim do Instituto de Geociências da USP, Série Científica, 18:67-83.

Lima, M.R.; Fulfaro, V.J. \& Bartorelli, A. 1980. Análise palinológica de sedimentos cretáceos da região de Marabá, Estado do Pará. Boletim do Instituto de Geociências da USP, 11:155-161.

Limarino, C.O.; Passalia, M.G.; Llorens, M.; Vera, E.; Perez Loinaze, V.S. \& Cesari, S. 2012. Depositional environments and vegetation of Aptian sequences affected by volcanism in Patagonia. Palaeogeography, Palaeoclimatology, Palaeoecology, 323325:22-41. doi: 10.1016/j.palaeo.2012.01.021.

Llorens, M. 2008. Estudio palinológico de la Formación Punta del Barco, Cretácico Inferior (Aptiano) de la provincia de Santa Cruz, Argentina. Descripciones sistemáticas II: esporas triletes apiculadas, murornadas, tricrasadas y perinadas. Implicancias paleoecológicas. Ameghiniana, 45:273-278.

Mahmoud, M.S.; Soliman, H.A. \& Deaf, A.S. 2007. Early Cretaceous (Aptian-Albian) palynology of the Kabrit-1 borehole, onshore Northern Gulf of Suez, Egypt. Revista Española de Micropaleontología, 39:169-187.

McNeill, J.; Barrie, F.R.; Buck, W.R.; Demoulin, V.; Greuter, W.; Hauwksworth D.L.; Herendeen, P.S.; Knapp, S.; Marhold, K.; Prado, J.; Prud'Homme Van Reine, W.S.; Smith, G.S.; Wiersema, J.H. \& Turland, N.J. (eds.) 2012. International Code of Nomenclature for Algae, Fungi, and Plants (Melbourne Code). Koenigstein, Koeltz Scientific Books (Regnum Vegetabile 154).

Medina, F.; Archangelsky, S.; Guler, V.; Archangelsky, A. \& Cárdenas, O. 2008. Estudio bioestratigráfico integrado del perfil La Horqueta (límite Aptiano-Albiano), Lago Cardiel, Patagonia, Argentina. Revista del Museo Argentino de Ciencias Naturales, 10:273-289.

Narvaez, P.L.; Mego, N. \& Pramparo, M.B. 2013. Cretaceous cicatricose spores from north and central-western Argentina: taxonomic and biostratigraphic discussion. Palynology, 37:202217. doi: $10.1080 / 01916122.2012 .762062$

Narváez, P.L. 2009. Palinoestratigrafia, paleoambientes y cambios climáticos durante el Cretácico final y Paleógeno de la Cuenca del Grupo Salta, República Argentina. Universidad Nacional de Cuyo, Tesis Doctoral, 229 p.

Paden Phillips, P. \& Felix, C.J. 1971. A study of Lower and Middle Cretaceous spores and pollen from the Southeastern United States. I. Spores. Pollen et Spores, 13:280-348.

Playford, G. 1971. Palynology of Lower Cretaceous (Swan River) strata of Saskatchewan and Manitoba. Palaeontology, 14:533-565.
Potonié, R. 1956. Synopsis der Gattungen der Sporae Dispersae. I Teil: Sporites. Beihefte zum Geologischen Jahrbuch, 23:1-103.

Potonié, R. \& Kremp, G. 1954. Die Gattungen der paläozoischen Sporae Dispersae und ihre Stratigraphie. Geologische Jahrbuch, 69:111-194.

Prámparo, M.B. 1988. Esporas triletes levigadas y apiculadas de la Formación La Cantera (Cretácico de la Cuenca de San Luis) en su localidad tipo. In: CONGRESO ARGENTINO DE PALEONTOLOGÍA Y BIOESTRATIGRAFÍA, 4, 1986. Actas, Mendoza, v. 3, p. 51-62.

Prámparo, M.B. 1989. Las esporas de Schizaeaceae (Cicatricosisporites y Appendicisporites) del Cretácico Inferior, Cuenca de San Luis, Argentina. Revista Española de Micropaleontologia, 21:355-372.

Prámparo, M.B. 1990. Palynostratigraphy of the Lower Cretaceous of the San Luis Basin, Argentina. Its place in the Lower Cretaceous floral provinces pattern. Neues Jahrbuch für Geologie und Paläontologie, Abh, 181:247-258.

Prámparo, M.B. 1994. The Lower Cretaceous palynoflora of the La Cantera Formation, San Luis Basin: correlation with other Cretaceous palynofloras of Argentina. Cretaceous Research, 15:193-203. doi: 10.1006/cres.1994.1010

Prámparo, M.B. 1999. Microfitoplancton orgánico del Cretácico Inferior de la Cuenca de San Luis. Parte I: Scenedesmaceae y Chlorococcaceae. Buenos Aires, Asociación Paleontológica Argentina, p. 39-42 (Publicación Especial 6).

Prámparo, M.B.; Ballent, S.C.; Gallego, O.F. \& Milana, J.P. 2005. Paleontología de la Formacion Lagarcito (Cretácico inferior) en la provincia de San Juan, Argentina. Ameghiniana, 42:93-114.

Prámparo, M.B.; Quattrocchio, M.; Gandolfo, M.A.; Zamaloa, M.del C. \& Romero E. 2007. Historia evolutiva de las angiospermas (Cretácico-Paleógeno) en Argentina a través de los registros paleoflorísticos. Buenos Aires, Asociación Paleontológica Argentina, p. 157-172 (Publicación Especial 11).

Pryer, K.M.; Schuettpelz, E.; Wolf, P.G.; Schneider, H.; Smith, A.R. \& Cranfill, R. 2004. Phylogeny and evolution of ferns (monilophytes) with a focus on the early leptosporangiate divergences. American Journal of Botany, 91:1582-1598. doi:10.3732/ajb.91.10.1582

Puebla, G.G. 2009. Nuevo morfotipo de hoja de angiosperma del Cretácico Temprano (Aptiano Tardío), Cuenca de San Luis, Argentina. Ameghiniana, 46:557-566.

Puebla, G.G.; Mego, N. \& Prámparo, M.B. 2012. Asociación de briofitas de la Formación La Cantera, Aptiano tardío, Cuenca de San Luis, Argentina. Ameghiniana, 49:217-229. doi: 10.5710/ AMGH.v49i2(504)

Quattrocchio, M.E.; Martínez, M.A.; Carpinelli Pavicich, A. \& Volkheimer, W. 2006. Early Cretaceous palynostratigraphy, palynofacies and palaeoenvironments of well sections in northeastern Tierra del Fuego, Argentina. Cretaceous Research, 27:584-602. doi.org/10.1016/j.cretres.2005.11.012

Regali, M.S.P.; Uesugui, N. \& Santos, A.S. 1974. Palinologia dos sedimentos meso-cenozoicos do Brasil. Boletim Técnico da Petrobrás, 17:263-301.

Regali, M.S.P. \& Viana, C.F. 1989. Sedimentos do NeojurássicoEocretácico do Brasil: Idade e Correlação com a Escala Internacional. Rio de Janeiro, Petrobrás, 95 p.

Rivarola, D. \& Spaletti, L. 2006. Modelo de sedimentación continental para el rift cretácico de la Argentina Central. Ejemplo de la Sierra de las Quijadas, San Luis. Revista de la Asociación Geológica Argentina, 61:63-80. 
Scott, L. 1976. Palynology of Lower Cretaceous deposits from the Algoa Basin (Republic of South Africa). Pollen et Spores, 18:563-609.

Schrank, E. \& Ibrahim, M.I. 1995. Cretaceous (Aptian-Maastrichtian) palynology of foraminifera-dated wells (KRM-1, RG-18) in northwestern Egypt. Berliner Geowissenschafliches Abhandlundgen, 120:169-182.

Singh, C. 1964. Microflora of the Lower Cretaceous Mannville Group, East-Central Alberta. Research Council of Alberta, Bulletin 15:1-239.

Singh, C. 1971. Lower Cretaceous microfloras of the Peace River Area, Northwestern Alberta. Research Council of Alberta, Bulletin 28:1-299.

Smith, A.V.H. \& Butterworth, M.A. 1967. Miospores in the coal seams of the Carboniferous of Great Britain. Special Papers in Palaeontology, 1:1-324.

Smith, A.R.; Pryer, K.M.; Schuettpelz, E.; Korall, P.; Schneider, H. \& Wolf, P.G. 2006. A classification for extant ferns. Taxon, 55:705-731.

Srivastava, S.K. 1975. Microspores from the Fredericksburg Group (Albian) of the southern United States. Paleobiologie Continentale, 6:1-119.

Srivastava, S.K. 1981. Stratigraphic ranges of selected spores and pollen from Fredericksburg Group (Albian) of southern United States. Palynology, 5:1-27. doi:10.1080/01916122.1 981.9989215

Vallati, P. 2001. Middle Cretaceous microflora from the Huincul Formation ("Dinosaurian Beds") in the Neuquen Basin, Patagonia, Argentina. Palynology, 25:179-197. doi:10.1080/0 1916122.1981.9989215

Venkatachala, B.S.; Kar, R.K. \& Raza, S., 1968. Palynology of the Mesozoic sediments of Kutch, W. India-3. Morphological study and revision of the spore genus Trilobosporites Pant ex Potonié 1956. The Palaeobotanist, 17:123-126.
Volkheimer, W. 1972. Estudio palinológico de un carbón caloviano de Neuquén y consideraciones sobre los paleoclimas jurásicos de la Argentina. Revista del Museo de La Plata, Paleontología, 6:101-157.

Volkheimer, W. 1974. Palinologia estratigráfica del Jurásico de la Sierra de Chacai Có y adyacencias (Cuenca Neuquina). II. Descripción de los palinomorfos del Jurásico inferior y Aaleniano (Formaciones Chacai Có y Los Molles). Ameghiniana, 11:135-172.

Volkheimer, W. \& Melendi, D. 1976. Palinomorfos como fósiles guía. Tercera parte: Técnicas de laboratorio palinológico. Revista Minera de Geología y Mineralogía, 34:19-30.

Volkheimer, W. \& Salas, A. 1976. Estudio palinológico de la Formación Huitrín, Cretácico de la Cuenca Neuquina, en su localidad tipo. In: CONGRESO GEOLÓGICO ARGENTINO, 6, 1975. Actas, Bahía Blanca, v. 1, p. 433-456.

Volkheimer, W.; Caccavari, M. \& González-Amicón, O.R. 1981. Estudio palinológico de estratos Liásicos en el borde austral de la Cuenca Neuquina. In: CONGRESO GEOLÓGICO ARGENTINO, 8, 1981. Actas, San Luis, Asociación Geológica Argentina, v. 4, p. 777-793.

Volkheimer, W.; Gallego, O.; Cabaleri, N.; Armella C.; Narváez, P.; Silva-Nieto, D.G. \& Paez, M.A. 2009. Stratigraphy, palynology, and conchostracans of a Lower Cretaceous sequence at the Cañadon Calcareo locality, Extra-Andean central Patagonia: age and palaeoenvironmental significance. Cretaceous Research, 30:270-282. doi: 10.1016/j.cretres.2008.07.010

Yrigoyen, M. 1975. La edad Cretácica del Grupo del Gigante (San Luis) y su relación con cuencas circunvecinas. In: CONGRESO GEOLÓGICO ARGENTINO DE PALEONTOLOGÍA Y BIOESTRATIGRAFÍA, 2, 1975. Actas, Buenos Aires, v. 2, p. $29-56$.

Received in March, 2013; accepted in November, 2013.

Apéndice 1. Lista de las esporas triletes identificadas durante este estudio, ordenadas alfabéticamente y con referencia a su número de ilustración.

Appendix 1. Alphabetical list of the trilete spores identified during this study with the illustration number pointed.

Concavissimisporites penolaensis Dettmann, 1963 (Figuras 2A,B)

Concavissimisporites punctatus (Delcourt \& Sprumont) Brenner, 1963 (Figura 2C)

Concavissimisporites variverrucatus (Couper) Brenner, 1963 (Figuras 2D,E)

Concavissimisporites sp. (Figura 2F)

Converrucosisporites cf. C. exquisitus Singh, 1971 (Figura $2 \mathrm{G}$ )

Impardecispora apiverrucata (Couper) Venkatachala, Kar \& Raza, 1968 (Figuras 2H-J)

Impardecispora texensis Srivastava, 1975 (Figuras 2K,L)

Impardecispora sp. (Figuras 3A-C)

Leptolepidites major Couper, 1958 (Figura 3D)

Leptolepidites verrucatus Couper, 1953 (Figuras 3E-G)

Reyrea polymorphus Herngreen, 1973 (Figura 3H)

Verrucosisporites rotundus Singh, 1964 (Figuras 3I,J)

Verrucosisporites varians Volkheimer, 1972 (Figura 3K) 